\title{
Mis primeros 100 colgajos libres
}

\author{
Patricio Andrades C. ${ }^{1}$, Carlos Domínguez C. ${ }^{1}$, Juan Pablo Cisternas V. ${ }^{1}$, \\ Stefan Danilla E. ${ }^{1}$, Cristian Erazo C. ${ }^{1}$ y Sergio Sepúlveda P. ${ }^{1}$
}

\section{My first 100 free flaps}

Introduction: Microsurgical techniques have been consolidated as a safe and reproducible reconstructive strategy with high success rates. They allow to successfully solve complex coverage defects. Its development has been driven by technological improvements, technical standardization and perioperative management. The development and implementation of microsurgery is a complex process, requiring a progressive work by the health team. Objective: Show experience accumulated by the main author in reconstructive surgery during his first 100 free flap and the technical changes due to the modernization. Material and Methods: Retrospective series of 100 free flaps performed by the lead author during the years 2010 to 2015. All patients submitted to reconstructive microsurgery with free flaps were included and no exclusion criteria were established. Clinical and surgical history were reviewed. Descriptive statistics were used to present the results. Results: 100 free flaps, $62 \%$ of male patients, mean age $58 \pm 15$ years. The most used fins were fasciocutaneous (77\%), in particular the anterolateral flap of the thigh (47\%). Head and neck defects $(47 \%)$ were the most frequent defects. There were 10 vascular complications (6 arterial and 4 venous), with a failure rate of $7 \%$. Discussion: Teamwork, the use of horse flaps and new technologies are the cornerstone of the microsurgery experience presented. This corresponds to the continuation of the line of work sustained over time.

Key words: microsurgery, free flap, plastic surgery.

\section{Resumen}

Introducción: Las técnicas reconstructivas microquirúrgicas se han consolidado como una estrategia segura, reproducible y con altas tasas de éxito que permiten dar solución a defectos de cobertura complejos. Su desarrollo se ha impulsado por mejoras tecnológicas, estandarización de la técnica y manejo perioperatorio. El desarrollo e implementación de la microcirugía es un proceso complejo, que requiere de un trabajo progresivo por parte del personal de salud. Objetivo: Presentar la experiencia acumulada por el autor principal en cirugía reconstructiva utilizando sus 100 primeros colgajos libres y los cambios de la técnica producto de la modernización. Material y Métodos: Serie de casos retrospectiva de colgajos libres consecutivos realizados por el autor principal, durante los años 2010 al 2015. Se incluyó a todos los pacientes sometidos a cirugía reconstructiva mediante colgajos microquirúrgicos, no estableciéndose criterios de exclusión. Se revisaron antecedentes clínicos y quirúrgicos, utilizando estadística descriptiva para presentar los resultados. Resultados: 100 colgajos libres, $62 \%$ pacientes de sexo masculino, edad promedio de $58 \pm 15$ años. Los colgajos más utilizados fueron fasciocutáneos (77\%), en particular el colgajo antero-lateral de músculo (47\%), siendo los defectos de cabeza y cuello (47\%) los más frecuentes. Se observaron 10 complicaciones vasculares de los colgajos (6 arteriales y 4 venosas), con una tasa de pérdida total de 7\%. Discusión: El trabajo en equipo, uso de colgajos regalones y utilización de nuevas tecnologías son los pilares de la experiencia microquirúrgica presentada. Esta corresponde a la continuación de una línea de trabajo sostenida en el tiempo.

Palabras clave: microcirugía, colgajo libre, cirugía plástica. 


\section{Introducción}

La historia de la cirugía reconstructiva está marcada por el desarrollo y mejora de estrategias reconstructivas para dar solución a defectos tisulares cada vez más complejos, buscando optimizar los resultados funcionales y estéticos, además de disminuir las secuelas del sitio donante ${ }^{1}$. Durante las últimas dos décadas las técnicas reconstructivas microquirúrgicas se han consolidado como una estrategia reconstructiva segura, reproducibles y con tasas de pérdida que fluctúan entre $1-14 \%$, según series internacionales ${ }^{2,3}$. Su desarrollo se ha visto impulsado por mejoras tecnológicas en equipamiento, instrumental, estandarización de técnica y manejo perioperatorio, con lo que se ha logrado utilizar en pacientes con defectos de cobertura cada vez más complejos ${ }^{4,5}$. Diversas series describen su uso en pacientes oncológicos con extensas resecciones en cabeza y cuello ${ }^{6-8}$, heridas traumáticas de extremidades con defectos extensos o exposición de material de osteosíntesis ${ }^{9}$, así como reimplante de miembros amputados $^{10,11}$. Recientemente se ha perfilado como una estrategia constructiva en la cirugía de cambio de $\operatorname{sexo}^{12,13}$ y como pilar fundamental en los procedimientos de alotrasplante de tejidos compuestos ${ }^{14}$.

El desarrollo e implementación de la microcirugía es un proceso complejo, que requiere de un trabajo progresivo por parte del personal de salud, tanto para la adquisición de destrezas quirúrgicas de los cirujanos como para el desarrollo de un plan de trabajo sistematizado ${ }^{15,16}$. Este proceso se condice con la capacidad de dar respuesta cada vez a problemas más difíciles de solucionar. Sepúlveda et $\mathrm{al}^{3}$ presentó la primera experiencia a nivel nacional, iniciada en el Hospital Barros Luco Trudeau y continuada en el Hospital Clínico de la Universidad de Chile. En concordancia con lo anterior, el objetivo de este trabajo es presentar una serie clínica que representa la experiencia acumulada por el autor principal (PA) en el desarrollo de una línea de trabajo sostenida en el tiempo en el área de reconstrucción con colgajos libres, presentando los primeros 100 casos y los cambios de la técnica producto de su modernización.

\section{Material y Métodos}

Presentamos una serie de casos retrospectiva, constituida por los primeros 100 colgajos libres consecutivos realizados por el autor principal (PA), durante los años 2010 al 2015 en el Hospital Clínico de la Universidad de Chile y Hospital del Trabajador de Santiago. Se incluyó a todos los pacientes sometidos a cirugía reconstructiva mediante colgajos microquirúrgicos en forma sucesiva, no estableciéndose criterios de exclusión. Se revisaron las fichas clínicas y protocolos operatorios para recolectar los siguientes datos: edad del paciente, comorbilidad, indicación de la microcirugía, localización del defecto, colgajo utilizado, datos de la anastomosis, presencia de trombosis arterial y/o venosa y otras complicaciones asociadas a corto y largo plazo. Se definió falla total como aquella situación en la cual el colgajo evoluciona a necrosis total, ya sea por trombosis arterial, venosa o ambas, pese a uno o más intentos de rescate. Se definió rescate como aquellos casos en los cuales se detectó falta de perfusión del colgajo debiendo reintervenir al paciente tempranamente para rehacer en forma exitosa o fallida las anastomosis arteriales $\mathrm{y} / \mathrm{o}$ venosas.

Los pacientes considerados para reconstrucción con colgajos microquirúrgicos presentaban defectos de cobertura cutánea, donde opciones reconstructivas con colgajos locales o regionales eran insuficientes, habían sido utilizadas previamente $\mathrm{u}$ ofrecían resultados con secuelas funcionales y estéticas significativas. Los pacientes debían tener como requisito estar con sus patologías de bases estabilizadas, en buenas condiciones generales para resistir una gran cirugía y un estudio preoperatorio completo. Se realizó estudio vascular dirigido de los vasos receptores. En extremidad inferior, los vasos receptores se evaluaron clínicamente buscando pulsos y utilizando Eco Doppler y/o Angio TC. En los casos de reconstrucción de extremidad inferior la angiografía convencional se realiza cuando existe discordancia entre los hallazgos clínicos (pulsos distales) y el Angio TC. En cabeza, cuello y tronco se realizó estudio de vasos receptores con Angio TC en casos de territorios previamente manipulados. Dependiendo del colgajo a utilizar se realizó estudio del pedículo vascular con Angio TC usando protocolo de marcación de perforantes previamente publicado ${ }^{17}$. En el caso de colgajo radial solo se realizó la evaluación clínica de competencia vascular del arco palmar con test de Allen ${ }^{18}$.

La realización de un colgajo libre presentó 4 tiempos, en el cual cada miembro del equipo quirúrgico posee un rol definido, permitiendo armonizar el desarrollo de este procedimiento. La cirugía comienza con el tiempo resectivo en el caso de lesiones oncológicas o la preparación de la zona receptora en el caso de heridas con defectos de cobertura, identificando y preparando los vasos receptores. En paralelo, una vez definido el tamaño del defecto, se diseña y eleva el colgajo a utilizar, tomando en 
consideración el tamaño del defecto, longitud del pedículo vascular, función a desempeñar (cobertura cutánea, aportar volumen, soporte estructural, etc.) y concordancia cutáneo. Previo a la sección del pedículo vascular del colgajo se probó la vitalidad de éste, evaluándose su irrigación. Previo a la realización de la anastomosis se realizó el montaje del colgajo, permitiendo determinar la mejor orientación de éste y del pedículo. Si bien existen diversas técnicas descritas para la confección de la anastomosis vascular, en esta serie la anastomosis arterial se realizó con Nylon 9/0 o 10/0 a puntos separados o sutura corrida, y en el caso de la anastomosis venosa se utilizó sutura mecánica "Coupler" (2,0$3,5 \mathrm{~mm})^{19}$. Concluida la anastomosis, se reevaluó la perfusión del colgajo observando principalmente el sangrado de bordes y el llene capilar. Finalizada la anastomosis se completa el montaje del colgajo tomando siempre en consideración la ubicación, disposición del pedículo vascular y anastomosis. Esta etapa presenta un grado de dificultad variable, dependiendo de la ubicación y función que deba cumplir el colgajo. Desde casos simples como la cobertura de un defecto cutáneo en la región esternal, hasta casos más complejos como la reconstrucción de la vía aérea digestiva superior en un tumor de faringe. Durante este proceso se realizó en forma paralela el manejo de la zona dadora buscando un cierre adecuado.

Concluida la cirugía todos los pacientes cursaron al menos $24 \mathrm{~h}$ de observación en Unidad de Cuidados Intermedios con monitorización clínica seriada del paciente y del colgajo (color, temperatura, llene capilar y sangrado) por parte del personal de enfermería. En forma complementaria se realizó monitorización mediante el uso de eco al pedículo vascular y recientemente se ha incorporado el uso de Smartphone con su función de cámara para mantener un registro de la evolución clínica.

\section{Resultados}

La serie considera los 100 primeros colgajos libres realizados en forma consecutiva por el autor principal (Tabla 1). Del total, 62 pacientes fueron de sexo masculino $(62 \%)$, con una edad promedio de $58 \pm 15$ [6-76] años. Los colgajo más utilizados fueron los colgajos fasciocutáneos en 77 (77\%) casos, en particular el colgajo antero-lateral de músculo (ALT) en 47 (47\%) casos, seguido por el colgajo radial en $22(22 \%)$ casos. Se realizaron $15(15 \%)$ colgajos musculares y $8(8 \%)$ colgajos óseos (Figura $1)$. Los defectos de cobertura más frecuentes se ubicaron en cabeza y cuello (47\%) y en extremidades inferiores $(40 \%)$. Las etiologías que condicionaron estas cirugías reconstructivas correspondieron a reconstrucciones posresección oncológica oncológicas en $55 \%$ y defectos traumáticos en $45 \%$ de los casos.

La serie presenta 10 complicaciones vasculares de los colgajos ( 6 arteriales y 4 venosas). En cuatro de estos casos no se realizó reexploración debido a las dudas en la viabilidad del colgajo en 2 casos y debido a las malas condiciones del paciente en otros

Tabla 1. Tabla resumen de casos incluidos

\begin{tabular}{|c|c|c|c|c|c|c|c|c|c|}
\hline & Total & EEII & EESS & $\begin{array}{l}\text { Cabeza } \\
\text { y cuello }\end{array}$ & Mama & $\begin{array}{c}\text { Trombosis } \\
\text { arterial }\end{array}$ & $\begin{array}{c}\text { Trombosis } \\
\text { venosa }\end{array}$ & $\begin{array}{c}\text { Pérdida } \\
\text { total }\end{array}$ & Otras complicaciones \\
\hline ALT & 47 & 29 & 3 & 15 & 0 & 3 & 2 & 5 & $\begin{array}{l}1 \text { seroma crónico } \\
32 \text { dehiscencias ZD } \\
3 \text { rescates fallidos }\end{array}$ \\
\hline Radial & 22 & 0 & 0 & 22 & 0 & 1 & 0 & 0 & $\begin{array}{l}2 \text { fístulas salivales } \\
1 \text { rescate exitoso }\end{array}$ \\
\hline Recto abdominal & 7 & 3 & 0 & 4 & 0 & 1 & 1 & 1 & 1 rescate fallido \\
\hline Fíbula & 6 & 0 & 1 & 4 & 0 & 0 & 0 & 0 & $\begin{array}{l}1 \text { dehiscencia ZD } \\
1 \text { exposición oral reop. }\end{array}$ \\
\hline Escápula & 2 & 0 & 0 & 2 & 0 & 0 & 0 & 0 & 1 fístula salival \\
\hline
\end{tabular}

ALT: Colgajo Antero Lateral de muslo, DIEP: Colgajo perforante de arteria epigástrica profunda inferior, EEII: Extremidades Inferiores, EESS: Extremidades superiores, ZD: Zona dadora. 
Figura 1. Distribución de colgajos utilizados. ALT: de muslo; DIEP: Colgajo perforante de arteria epigástrica profunda inferior. Colgajo Antero Lateral

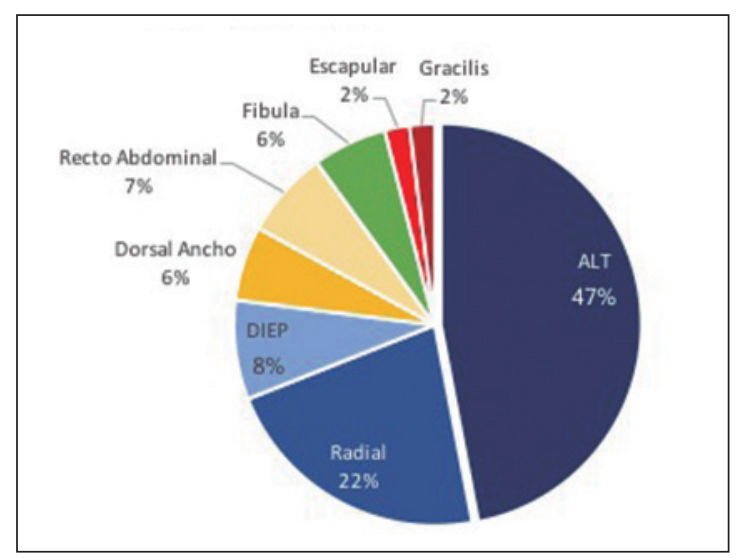

2 casos. En 6 casos si se reexploró las anastomosis microvasculares, lográndose un rescate exitoso en 3 casos (50\% de éxito). Finalmente, la serie presenta una tasa de pérdida total de colgajos de un $7 \%$. En 2 de estos casos se realizó un segundo colgajo libre que fue exitoso. En un paciente con defecto en tercio distal de pierna se le realizó un colgajo libre de gracilis después de fallar un colgajo ALT. En el segundo caso se realizó con éxito la reconstrucción de un gran defecto de tercio medio e inferior de la cara con un colgajo libre musculocutáneo de dorsal ancho tras haber fallado un colgajo de recto abdominal. En los restantes 5 colgajos libres fallidos se utilizaron otras alternativas no microquirúrgicas para resolver el problema. Una paciente requirió un colgajo dorsal ancho para reconstruir su mama tras falla de un colgajo DIEP. Dos pacientes diabéticos fueron a amputación dado el avance de su patología

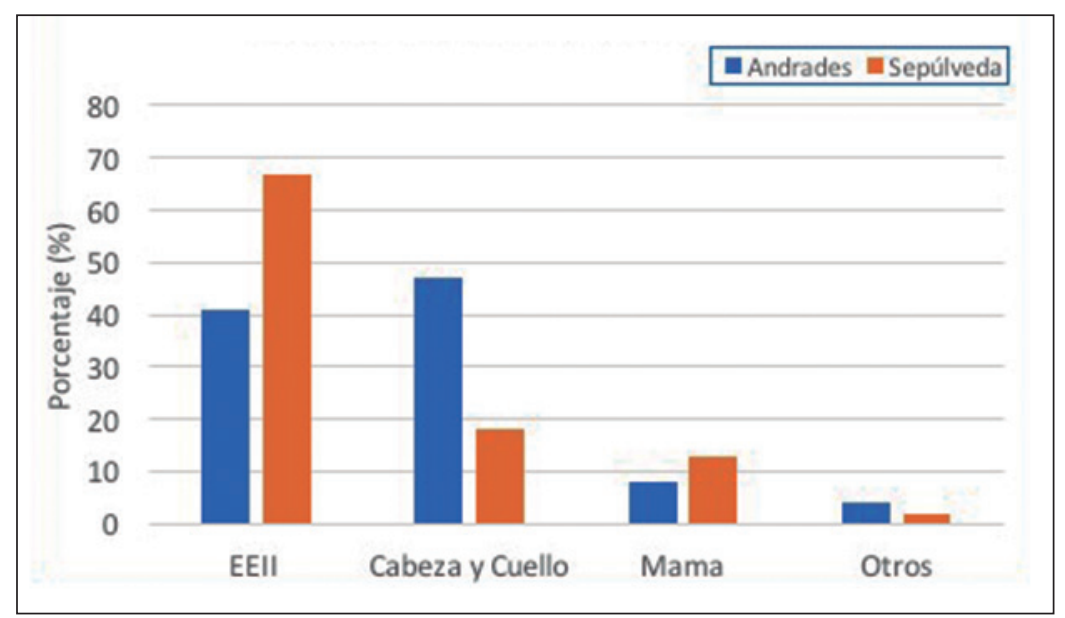

Figura 2. Comparación de zonas a reconstruir utilizando colgajos libres. de base, y en 3 casos de reconstrucción de extremidad inferior se realizaron colgajos locales e injertos de piel parcial para lograr cobertura de sus defectos.

Se observó la presencia de otras 45 complicaciones en la serie. El colgajo ALT presentó en zona dadora 32 dehiscencias que se manejaron en forma conservadora. Un paciente con prótesis de fémur cubierta por un ALT, presentó un seroma crónico secundario a recidiva de su enfermedad de base requirió una amputación a los 2 años después de su cirugía de cobertura. El colgajo radial presentó en la zona dadora, pérdidas parciales del injerto en 3 casos que solo requirieron curaciones para su cierre. Se observaron 2 fístulas salivales tras reconstrucción de hipofaringe con colgajo radial. Un paciente respondió a tratamiento conservador y otro paciente requirió un colgajo musculocutáneo de pectoral mayor para su cierre. Tres pacientes desarrollaron un seroma en la zona dadora del colgajo de dorsal ancho que respondieron a punciones seriadas. Un paciente con colgajo de músculo gracilis requirió injertos complementarios sobre el músculo en la zona receptora. Lo mismo ocurrió en la zona dadora de un paciente con colgajo de peroné que requirió injertos complementarios en zona dadora. Otro paciente con colgajo de peroné presentó exposición de material de OTS intraoral que tuvo que ser reoperado para su remoción. Finalmente, un paciente con colgajo escapular para reconstrucción de mandíbula presentó una fístula salival que respondió adecuadamente a tratamiento médico.

\section{Discusión}

La serie presentada representa la continuación de trabajo de más de 35 años de dedicación y desarrollo de la microcirugía en nuestro centro. Al comparar nuestra serie con la publicada por Sepúlveda et $\mathrm{al}^{3}$, se evidencia claramente un cambio en el perfil de las necesidades y estrategias reconstructivas. En la serie anterior se observa un predominio de colgajos libres realizados en extremidades inferiores $(67 \%)$, producto de lesiones traumatológicas, seguido por $18 \%$ lesiones de cabeza y cuello. Al contrario de lo presentado en esta serie donde se observa un aumento del número de colgajos libres en cabeza y cuello $(47 \%)$ y una disminución en extremidades inferiores, explicado por el desarrollo de una línea de trabajo multidisciplinario (otorrinolaringología, cirugía de cabeza y cuello, cirugía maxilofacial) en el manejo de estas lesiones (Figura 2). Otra diferencia significativa está dada por el tipo de colgajos utilizados, basada inicialmente en el uso de colgajos mus- 
culares (59\%), en la experiencia actual, se observa un aumento en el uso de colgajos fascio-cutáneos (77\%) y en particular los tipos perforantes (55\%), con una disminución de los colgajos musculares (15\%) (Figura 3). Lo cual es similar a la tendencia observada en otras series internacionales ${ }^{6,20}$. Todo lo anterior en el contexto de complicaciones vasculares y no vasculares semejantes entre ellas y a lo reportado en la literatura los últimos 20 años (Figura 4).

Klosterman et al ${ }^{21}$ describe el desarrollo de complicaciones generales entre un 37-53\%. Al analizar nuestras complicaciones (45\%) podemos decir que está dentro de lo aceptado, considerando que la gran mayoría de estas complicaciones fueron absolutamente menores y respondieron adecuadamente a tratamiento conservador. Series internacionales describen tasas de pérdida de colgajos del 1-14\% ${ }^{2}$, a nivel nacional la serie presentada por Sepúlveda reportó un $5 \%$ de pérdida ${ }^{3}$, menor al $7 \%$ observado en esta serie, lo cual se podría explicar en base a que esta experiencia considera todo el período de la curva de aprendizaje del autor.

La microcirugía moderna ha atravesado varias etapas en su desarrollo hasta llegar a ser lo que es hoy en día. Nuestra serie refleja esta modernización, la que, desde nuestro punto de vista está sustentada en 3 pilares fundamentales: el trabajo en equipo, el uso de colgajos regalones y el apoyo en nuevas tecnologías. El primer pilar que ha permitido el desarrollo de esta experiencia microquirúrgica corresponde a la conformación y consolidación de un trabajo en equipo. Un equipo tiene más conocimientos que cada individuo por separado lo que motiva a trabajar más y mejor y a minimizar las frustraciones. Esto ha permitido un manejo protocolizado de pacientes tanto en el estudio preoperatorio y control posoperatorio, permitiendo optimizar el manejo de los pacientes ${ }^{16,22,23}$. Además, la ejecución coordinada en pabellón de dos equipos quirúrgicos que permiten el trabajo en forma simultánea, tanto en la zona dadora y receptora, permite obtener una mayor eficiencia y optimizar los resultados. Desde nuestro punto de vista es uno de los elementos más importantes en la microcirugía moderna, no solo porque mejora todo el proceso reconstructivo, sino que también fortalece las relaciones de trabajo y la docencia.

En el último tiempo se ha observado un aumento en el número de colgajos libres descritos, principalmente por el desarrollo de colgajos perforantes dado por su versatilidad de diseño, longitud de pedículo, composición variable y menores complicaciones de zona dadora ${ }^{24}$. No obstante, la gran variedad de colgajos descritos en esta serie, al igual que lo

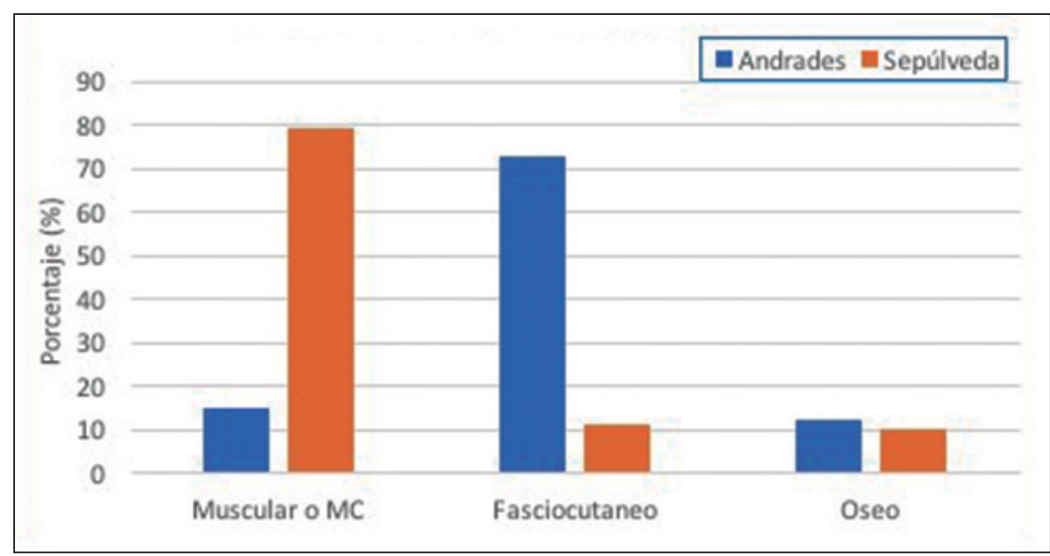

Figura 3. Comparación de tipo de colgajos utilizados. MC: Colgajo Músculo-Cutáneo.

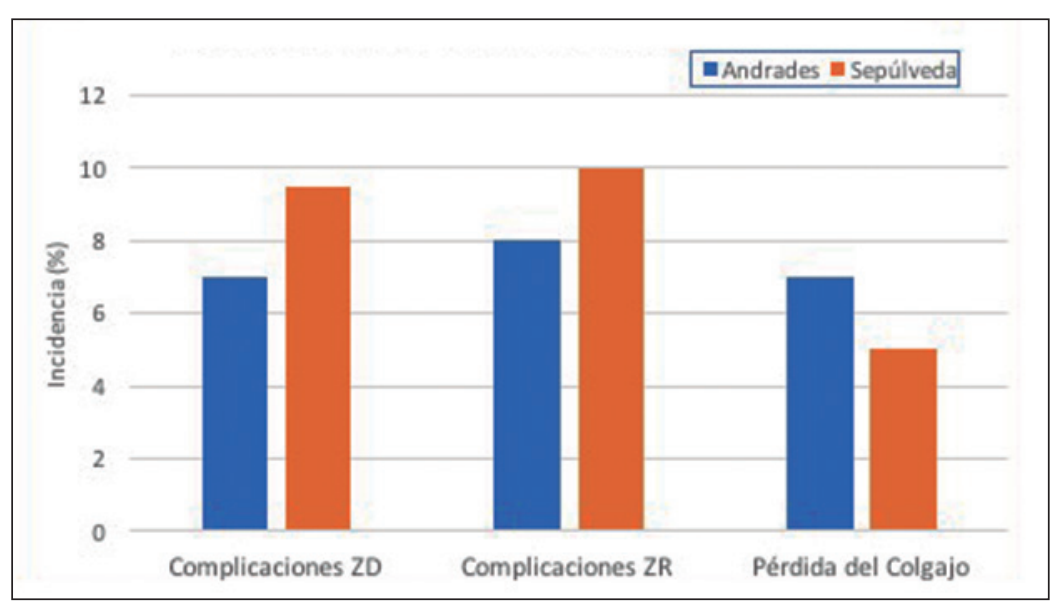

Figura 4. Comparación de complicaciones reportadas. ZD: Zona Dadora, ZR: Zona Receptora.

reportado por otros autores ${ }^{6,25}$, se observa el uso de un grupo limitado de colgajos (colgajos regalones o workhorse flaps) para dar respuesta a diferentes tipos defectos. El uso de colgajos regalones permite centrar la experiencia reconstructiva en un grupo limitado de colgajos con características particulares (fasciocutáneo, muscular y óseo) con el objeto de fortalecer las habilidades en torno a su diseño, elevación y manipulación, buscando disminuir las complicaciones. En nuestra serie se observa claramente el uso del colgajo regalones, siendo el colgajo ALT y radial los colgajos fasciocutáneo los más utilizados, el gracilis como colgajo muscular y el peroné y radial osteocutáneo como los colgajos óseos. Esto no significa estar desatento y cerrado a nuevas opciones de colgajos libres, sino solo que durante la primera etapa de la curva de aprendizaje se utilicen no más de 5 a 6 colgajos para dar solu- 
ción a la mayoría de los problemas reconstructivos ${ }^{26}$.

Durante las últimas décadas el desarrollo tecnológico ha permitido optimizar distintas etapas en la realización de un colgajo libre. El uso de Angio TC como parte del estudio vascular preoperatorio, ha permitido identificar y caracterizar el estado de vasos perforantes y receptores ${ }^{27}$. El desarrollo de un sistema estandarizado de marcación de perforantes, facilitando la elevación y diseño de los colgajos, disminuyendo los tiempos operatorios y complicaciones como ya lo hemos publicado previamente ${ }^{17}$. El uso de anastomosis venosa mecánica, mediante dispositivo Coupler, ha sido beneficioso en distintos aspectos, dado que junto con estandarizar el procedimiento, ha permitido disminuir los tiempos operatorios, acortando la curva de aprendizaje y manteniendo una baja tasa de complicaciones venosas ${ }^{19,28,29}$. El posoperatorio temprano ha demostrado ser el período durante el cual se desarrollan la mayor tasa de complicaciones asociadas a la microanastomosis y que son potencialmente reversibles ${ }^{30}$. El desarrollo y acceso a nuevas tecnologías han permitido mejorar las estrategias de monitorización de los colgajos. Tal es el caso del uso de eco doppler de superficie o implantado, que permiten evaluar el flujo vascular en el pedículo del colgajo. El doppler implantado se utiliza en el caso de colgajos "sumergidos", donde no es posible realizar una monitorización clínica ${ }^{31,33}$. El uso de imágenes termográficas ${ }^{34}$ permite evaluar la temperatura del colgajo y compararla en forma objetiva con la del resto del cuerpo. Además, el desarrollo y masificación del uso de los teléfonos inteligentes o Smart Phones con la capacidad para obtener y transmitir fotografías y vídeos, ha permitido mantener un mejor seguimiento de los pacientes $^{35}$. El verde de indocianina ${ }^{33}$ es una técnica que permite evaluar la perfusión tisular mediante el uso de un medio de contraste, presentando resultados promisorios, no obstante, no ha sido implementada en nuestro centro.
Esta serie representa el inicio de lo que esperamos sea un largo trabajo futuro intentando dar las mejores opciones reconstructivas a nuestros pacientes cada vez más demandantes y complejos. Refleja una experiencia actualizada de lo que es la reconstrucción microquirúrgica moderna y la continuación de lo que ya es una larga tradición de trabajo en el Equipo de Cirugía Plástica del Hospital Clínico de la Universidad de Chile.

\section{Responsabilidades éticas}

Protección de personas y animales. Los autores declaran que para esta investigación no se han realizado experimentos en seres humanos ni en animales.

Confidencialidad de los datos. Los autores declaran que han seguido los protocolos de su centro de trabajo sobre la publicación de datos de pacientes.

Derecho a la privacidad y consentimiento informado. Los autores han obtenido el consentimiento informado de los pacientes y/o sujetos referidos en el artículo. Este documento obra en poder del autor de correspondencia.

\section{Financiación}

Los autores declaran no haber recibido ninguna financiación para la realización de este trabajo.

\section{Conflicto de intereses}

Los autores declaran no tener ningún conflicto de intereses.

\section{Bibliografía}

1. Tamai S. History of Microsurgery. Plastic and Reconstructive Surgery. 2009;124:e282-94.

2. Xiong L, Gazyakan E, Kremer T, Hernekamp FJ, Harhaus L, Saint-Cyr M, et al. Free flaps for reconstruction of soft tissue defects in lower extremity: A metaanalysis on microsurgical outcome and safety. Microsurgery. 2016;36:511-24.
3. Sepúlveda S, De Carolis V, Andrades CP, Benítez S, Danilla ES, Erazo C, et al. Reconstrucción con colgajos libres: una experiencia de 33 años. Rev Chil Cir. 2013;65:502-8.

4. Andrades P, Bohannon IA, Baranano CF, Wax MK, Rosenthal E. Indications and outcomes of double free flaps in head and neck reconstruction. Microsurgery. 2009;29:171-7.

5. Roehl KR, Mahabir RC. A Practical
Guide to Free Tissue Transfer. Plastic and Reconstructive Surgery. 2013;132:147e-158e.

6. Wong CH, Wei FC. Microsurgical free flap in head and neck reconstruction. Eisele DW, editor. Head Neck. 2009;32:1236-45.

7. Liang J, Yu T, Wang X, Zhao Y, Fang $\mathrm{F}$, Zeng W, et al. Free tissue flaps in head and neck reconstruction: clinical application and analysis of 93 patients 
of a single institution. Brazilian Journal of Otorhinolaryngology. Associação Brasileira de Otorrinolaringologia e Cirurgia Cérvico-Facial. 2017;1-10. https://doi.org/10.1016/j.bjorl.2017.04.009

8. Andrades P, Militsakh O, Hanasono MM, Rieger J, Rosenthal EL. Current strategies in reconstruction of maxillectomy defects. Arch Otolaryngol Head Neck Surg. 2011;137:806,12.

9. Engel H, Lin C-H, Wei F-C. Role of Microsurgery in Lower Extremity Reconstruction. Plastic and Reconstructive Surgery. 2011;127:228S-238S.

10. Martínez E, Lagares A, Gacto P, Lorite P, Lobo F, Gómez I, et al. Reimplante de pulgar. Casuística del Hospital Universitario Virgen del Rocío (Sevilla) entre los años 2005-2010. Cirugía Plástica Ibero-Latinoamericana 2013;39:225-30.

11. Saint-Cyr M, Wong C, Buchel EW, Colohan S, Pederson WC. Free Tissue Transfers and Replantation. Plastic and Reconstructive Surgery. 2012 Dec;130:858e-878e.

12. Morrison SD, Shakir A, Vyas KS, Kirby J, Crane CN, Lee GK. Phalloplasty. Plastic and Reconstructive Surgery. 2016;138:594-615.

13. van der Sluis WB, Smit JM, Pigot GLS, Buncamper ME, Winters HAH, Mullender $\mathrm{MG}$, et al. Double flap phalloplasty in transgender men: Surgical technique and outcome of pedicled anterolateral thigh flap phalloplasty combined with radial forearm free flap urethral reconstruction. Microsurgery. 2017;43:215-7.

14. Pomahac B, Pribaz JJ, Bueno EM, Sisk GC, Díaz-Siso JR, Chandawarkar A, et al. Novel Surgical Technique for Full Face Transplantation. Plastic and Reconstructive Surgery. 2012;13:549-55.

15. Andrades CP, Erazo CC, Loo M, Roco H, Cabello R, Irarrazabal V. El laboratorio de microcirugía. Rev Chil Cir. 2005;57:36672.

16. Sylvester M, Roco H. Estableciendo protocolo de apoyo en microcirugía. Rev Chil Cir. 2015;67:545.

17. Andrades CP, Calderón M, Palavicino P, Danilla ES, Erazo CC, Benítez S, et al. Angio TC en la identificación preoperatoria de vasos perforantes. Rev Chil Cir. 2014;66:134-41.

18. Andrades CP, Elsa Calderon GM, Danilla ES, Benitez S, Erazo CC, Sepúlveda PS Colgajo radial: experiencia del equipo de Cirugía Plástica de la Universidad de Chile. Rev Chil Cir. 2011;63:459-67.

19. Andrades CP, Calderón ME, Danilla ES, Erazo CC, Benítez S, Sepúlveda P S. Microanastomosis venosa mecánica coupler $^{\circledR}$. Un importante aporte tecnológico a la microcirugía vascular. Rev Chil Cir. 2014;66:52-8.

20. Hallock G. A Paradigm Shift in Flap Selection Protocols for Zones of the Lower Extremity using Perforator Flaps. J Reconstr Microsurg. Thieme Medical Publishers; 2013;29:233-40.

21. Klosterman T, Siu E, Tatum S. Free Flap Reconstruction Experience and Outcomes at a Low-Volume Institution over 20 Years. Otolaryngol Head Neck Surg. 2015;152:832-7.

22. Patel UA, Hernández D, Shnayder Y, Wax MK, Hanasono MM, Hornig J, et al. Free Flap Reconstruction Monitoring Techniques and Frequency in the Era of Restricted Resident Work Hours. JAMA Otolaryngol Head Neck Surg. 2017;1-7. Published online June 1, 2017. doi:10.1001/jamaoto.2017.0304

23. Lamp S. Optimizing the Success of the Microvascular Free Flap. Plastic Surgical Nursing. 2013;33:86-7.

24. Man LX, Selber JC, Serletti JM. Abdominal Wall following Free TRAM or DIEP Flap Reconstruction: A MetaAnalysis and Critical Review. Plastic and Reconstructive Surgery. 2009;124:752-64.

25. Lutz BS, Wei F-C. Microsurgical Workhorse Flaps in Head and Neck Reconstruction. Clinics in Plastic Surgery. 2005;32:421-30.

26. Chang EI. My First 100 Consecutive Microvascular Free Flaps. Plastic and Reconstructive Surgery-Global Open. 2013;1:e27-8.

27. Ohkuma R, Mohan R, Baltodano PA, Lacayo MJ, Broyles JM, Schneider EB, et al. Abdominally Based Free Flap Planning in Breast Reconstruction with Computed Tomographic Angiography. Plastic and Reconstructive Surgery. 2014;133:483-94.

28. Medina ND, Fischer JP, Fosnot J, Serletti JM, Wu LC, Kovach SJ III. Lower Extremity Free Flap Outcomes Using an Anastomotic Venous Coupler Device. Annals of Plastic Surgery. 2014;72:176-9.

29. Pratt GF, Rozen WM, Westwood A, Hancock A, Chubb D, Ashton MW, et al. Technology-assisted and sutureless microvascular anastomoses: Evidence for current techniques. Microsurgery. 2011;32:68-76.

30. Zoccali G, Molina A, Farhadi J. Is long-term post-operative monitoring of microsurgical flaps still necessary? British Journal of Plastic Surgery. Elsevier Ltd; 2017;1-5. http://dx.doi.org/10.1016/j. bjps.2017.05.041

31. Rappoport D, Madrid A, Capdeville F, Valdés F. Utilidad de la monitorización de los colgajos microquirúrgicos. Rev Chil Cir. 2016;68:345-8.

32. Lohman R, Langevin CJ, Bozkurt M, Kundu N, Djohan RA Prospective Analysis of Free Flap Monitoring Techniques: Physical Examination, External Doppler, Implantable Doppler, and Tissue Oximetry. J Reconstr Microsurg. 2012;29:051-6.

33. Chae MP, Rozen WM, Whitaker IS, Chubb D, Grinsell D, Ashton MW, et al. Current Evidence for Postoperative Monitoring of Microvascular Free Flaps. Ann Plast Surg. 2015;74:621-32. doi: 10.1097/SAP.0b013e3181f8cb32

34. Just M, Chalopin C, Unger M, Halama D, Neumuth T, Dietz A, et al. Monitoring of microvascular free flaps following oropharyngeal reconstruction using infrared thermography: first clinical experiences. European Archives of OtoRhino-Laryngology. Springer Berlin Heidelberg; 2015:1-9.

35. Hee Hwang J, Mun G-H. An Evolution of Communication in Postoperative Free Flap Monitoring. Plastic and Reconstructive Surgery 2012;130:125-9. 